\title{
Time for a fresh look at ROP screening
}

Eye (2003) 17, 117-118. doi:10.1038/

sj.eye. 6700297

Compared to many medical conditions, retinopathy of prematurity (ROP) lends itself relatively easily to screening, for it is treatable and affects a defined population of preterm babies who are almost always resident in the hospital neonatal unit at the critical time. The report of an 11-year experience of ROP screening by Brennan et al in this issue of Eye is useful and thought provoking as it reflects maintained solid experience, confirms some aspects of the clinical features of this condition, but even more important, it highlights a number of the dilemmas of screening. The authors confirm the findings of other studies that ROP behaves in a relatively uniform manner, displays in its acute phase a high degree of interocular symmetry, and that severe disease requiring treatment develops over a relatively defined period that is best defined by postmenstrual age. ${ }^{1-3}$ While this last point is recognised by the current UK guidelines, ${ }^{4}$ the recommendation that screening starts at 6-7 weeks caused some pretty close shaves in the Newcastle study of Brennan et al as five babies needed treatment at the 7 th week. No ophthalmologist wants the fright of diagnosing ROP at such an advanced stage on the first examination, especially as there is a distinct possibility that in the future treatment will be undertaken at a slightly earlier stage. Brennan et al support the current birthweight (BW) and gestational age (GA) inclusion criteria of the UK guidelines ( $\leqslant 1500 \mathrm{~g}$ BW and $<32$ weeks

GA). They point out that if these were lowered to $\leqslant 1250 \mathrm{~g} \mathrm{BW}$ and $\leqslant 29$ weeks GA, as sometimes suggested to reduce the screening burden, then two babies included in their report would have been denied the opportunity for treatment.

Screening is currently performed by experienced ophthalmologists, who in the UK examine about 8200 babies to identify the $1.8 \%$
AR Fielder

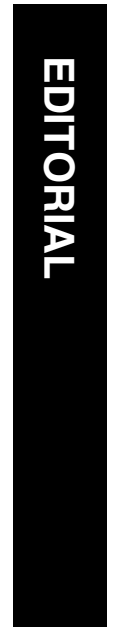

who require treatment. ${ }^{5}$ Brennan et al point out that each baby requires an average of 2.3 examinations and it takes an average of 39 examinations to detect each case of threshold ROP - not very dissimilar from the estimate of 55 examinations per threshold case obtained from a national audit. ${ }^{5}$ This low treatment yield represents a massive 'funnel effect', and it could be argued that this is not optimising skilled and expensive ophthalmic expertise, which might be better utilised for other more fruitful tasks. So, now is the time to seriously explore alternatives to screening by ophthalmologists. Transferring the screening role to the paediatrician falls into the category of passing the buck rather than real innovation, and cannot be robust using traditional examination techniques. However, the recent development of contact wide-field digital retinal imaging (eg RetCam 120) opens up exciting new opportunities for ROP screening and can utilise other staff such as a nurse or medical photographer. ${ }^{6,7}$ The images so obtained could either be subjected to full or partial grading (such as: no, mild or severe ROP) by the examiner, or transmitted (telemedicine) without preliminary analysis to the ophthalmologist for primary grading or for confirmation. Also images from babies suspected of harbouring serious diseases could also be shipped remotely for expert opinion. Brennan et al rightly point out that photographic screening must not result in deskilling ophthalmologists in the field. Clearly, research is required, but this new technology is here and it is important that we fully explore its potential, not only in the UK and other high-income countries, but also in middle-income countries where ROP-induced blindness is an even greater problem and ophthalmic expertise sparse. ${ }^{8}$

The recent report of a national UK audit showed that many ophthalmologists are making a great contribution to ROP screening, and frequently without this being recognised in their job plans. ${ }^{5}$ The report by Brennan et al in this
Department of

Ophthalmology Imperial College Faculty of Medicine 9th Floor Laboratory Block Charing Cross Hospital

Fulham Palace Road London W6 7RF, UK

\section{Correspondence:}

AR Fielder

Tel: 44 (0) 2083833693

Fax: 44 (0) 2083833651

E-mail: a.fielder@ic.ac.uk 
edition of Eye shows just how much work is required to identify one baby that needs treatment. This same group has also recently shown ${ }^{9}$ that the regionalisation of ROP services improves compliance with guidelines. So, now is the time to follow the excellent example of the Newcastle group to look hard at how we screen babies for ROP and to consider all aspects of service organisation, and promote concordance with clinicians and families, ${ }^{10}$ so that it is really both effective and efficient.

\section{References}

1 Fielder AR, Shaw DE, Robinson J, Ng YK. Natural history of retinopathy of prematurity: a prospective study. Eye 1992; 6: 233-242.

2 Quinn GE, Dobson V, Biglan A et al. The Cryotherapy for Retinopathy of Prematurity Cooperative Group. Correlation of retinopathy of prematurity in fellow eyes in the cryotherapy for retinopathy of prematurity study. Arch Ophthalmol 1995; 113: 469-473.

3 Palmer EA, Flynn JT, Hardy RJ et al. The Cryotherapy for Retinopathy of Prematurity Cooperative Group. Incidence and early course of retinopathy of prematurity. Ophthalmology 1991; 98: 1628-1640.
4 Report of a Joint Working Party of the Royal College of Ophthalmologists and British Association of Perinatal Medicine. Retinopathy of prematurity: guidelines for screening and treatment. Early Hum Dev 1996; 46: 239-258.

5 Haines L, Fielder AR, Pollock JI et al. Retinopathy of prematurity in the UK I: the organisation of services for screening and treatment. Eye 2002; 16: 33-38.

6 Schwartz SD, Harrison SA, Ferrone PJ, Trese MT. Telemedical evaluation and management of retinopathy of prematurity using a fiberoptic digital fundus camera. Ophthalmology 2000; 107: 25-28.

7 Roth DB, Morales D, Feuer WJ et al. Screening for retinopathy of prematurity employing the retcam 120: sensitivity and specificity. Arch Ophthalmol 2001; 119: 268-272.

8 Gilbert C, Rahi J, Eckstein M et al. Retinopathy of prematurity in middle-income countries. Lancet 1997; 350: $12-14$

9 Ziakas NG, Cottrell DG, Milligan DWA et al. Regionalisation of retinopathy of prematurity (ROP) screening improves compliance with guidelines: an audit of ROP screening in the Northern Region of England. Br J Ophthalmol 2001; 85: 807-810.

10 Aprahamian AD, Coats DK, Paysse EA, Brady-McCreery K. Compliance with outpatient follow-up recommendations for infants at risk for retinopathy of prematurity. JAAPOS 2000; 4: 282-286. 